Ann. Biol. anim. Bioch. Biophys., 1978, 18 (1), 29-32.

\title{
Etude de l'activité somatomédine et de l'hormone de croissance chez les sujets atteints de cirrhose alcoolique
}

\author{
par Rose-Marie SCHIMPFF*, D. LEBREC, M. DONNADIEU * \\ avec la collaboration technique de R. HAMELIN, CI. LASSARE
}

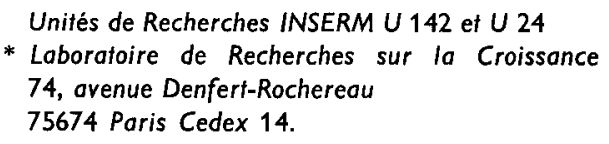

Summary. Somatomedin, growth hormone and cirrhosis.

Somatomedin activity and hGH levels were measured in blood samples taken simultaneously from the hepatic vein and the peripheral vein in 18 alcoholic patients with cirrhosis and in 10 adult patients without liver disease. In patients with cirrhosis, the somatomedin activity, measured in the hepatic vein, was significantly lower than in normal subjects $(P<0.001)$, although growth hormone levels were higher $(P<0.01)$. The hepatic blood flow was measured in 16 out of 28 subjects. In patients with cirrhosis a significant correlation was found between somatomedin activity and hepatic blood flow $(n=10$, $r=0.682, P<0.05)$.

\section{Introduction.}

Le rôle du foie sur l'activité somatomédine a été rapporté pour la première fois en 1970 par Mc Conaghey et Sledge. Le but de ce travail, effectué chez l'homme, est d'étudier les effets d'une maladie hépatique, la cirrhose alcoolique, sur le taux circulant de l'activité somatomédine (SM) du sérum et sur l'hormone de croissance plasmatique $(\mathrm{h} G \mathrm{H})$. La comparaison de l'activité des sangs prélevés simultanément dans une veine sus-hépatique (VSH) et dans une veine périphérique (VP) a permis de faire une estimation de la production hépatique de « l'activité $S M$ ».

\section{Sujets étudiés et méthodes.}

Dix huit adultes âgés de 34 à 66 ans, atteints de cirrhose alcoolique ont été étudiés. Chez 11 malades la cirrhose a été histologiquement confirmée. Chez tous les malades les prélèvements sanguins ont été effectués simultanément dans une VSH ef dans une VP, une heure après la mise en place des cathéters.

Dix sujets adultes considérés comme témoins, et indemnes de maladie hépatique et/ou endocrinienne, mais qui avaient dû subir un cathétérisme cardiaque, ont été étudiés dans les mêmes conditions. 
« L'activité SM » du sérum a été mesurée par l'incorporation de soufre radioactif $35 \mathrm{~S}$ dans le cartilage embryonnaire de poulet (Schimpff et Donnadieu 1973). L'hGH plasmatique a été mesurée par une technique radioimmunologique (Donnadieu et al., 1967). Le débit sanguin hépatique (DSH) a été mesuré au moyen de la méthode de la perfusion continue de vert d'indocyanine (Caesar et al., 1961). L'application du principe de Fick (Production $=\mathrm{DSH} \times$ Différence des concentrations) a permis d'estimer la production hépatique de « l'activité $S M$ » circulante.

Les analyses statistiques sont faites sur les séries appariées par le test de $t$ de Student-Fisher, et par les tests non paramétriques.

\section{Résultats.}

$$
\begin{array}{lc}
\text { Cirrhoses } & \text { Normaux } \\
(n=18) & (n=10)
\end{array}
$$

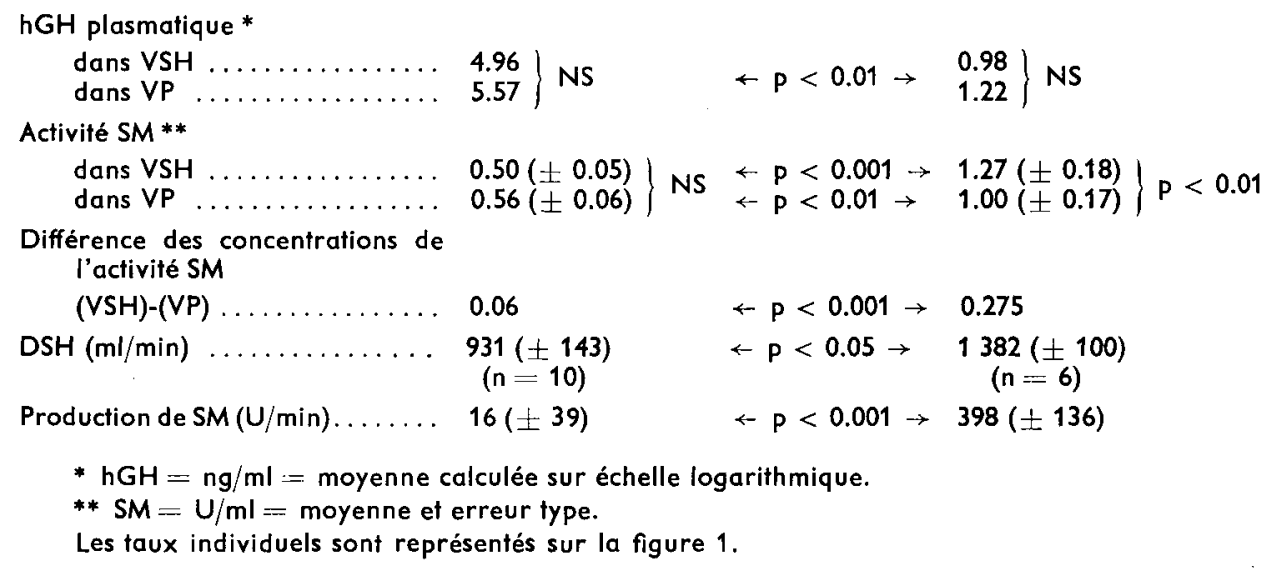

Chez les malades atteints de cirrhose alcoolique, il n'a pas été trouvé de corrélation entre « l'activité $S M$ » d'une part, et l'hormone de croissance, ou les tests biologiques d'insuffisance hépato-cellulaire évalués par l'albuminémie et le temps de Quick d'autre part. Une corrélation positive est trouvée entre « l'activité $S M$ » et le DSH $(r=0.682 p<0.05)$.

\section{Discussion.}

Le but de ce travail était de confirmer le rôle du foie sur la production de « l'activité $S M »$, dans des conditions physiologiques, alors que la plupart des éfudes ont été réalisées in vitro ou lors de perfusion in situ (Mc Conaghey et Sledge, 1970 ; Uthne et Uthne, 1972 ; Daughaday et al. 1976 ; Phillips et al., 1976). L'élévation de l'hGH ef la baisse de « l'activité $S M »$ chez les sujets atteints de cirrhose alcoolique ont été signalées par Wu et al. (1974) et par Lecornu (com. pers.). Ceci peut être retenu comme un argument indirect en faveur du rôle du foie sur « l'activité SM » circulante, bien qu'on ne puisse exclure, pour expliquer cette baisse, le rôle que peuvent jouer les mauvaises conditions nutritionnelles ou les troubles endocriniens particuliers à ces 
malades. L'originalité du travail présenté ici tient au protocole expérimental qui permet de mesurer « l'activité $S M$ » dans le sang sus-hépatique. On peut retenir alors comme arguments directs en faveur du rôle du foie : la différence observée chez le sujet normal entre « l'activité SM » du sang sus-hépatique et celle du sang périphérique, la corrélation positive qui existe, au cours de la cirrhose entre DSH et « activité $S M »$, enfin l'estimation de la production hépatique de Somatomédine, élevée chez le sujet normal et basse au cours de la maladie hépatique.
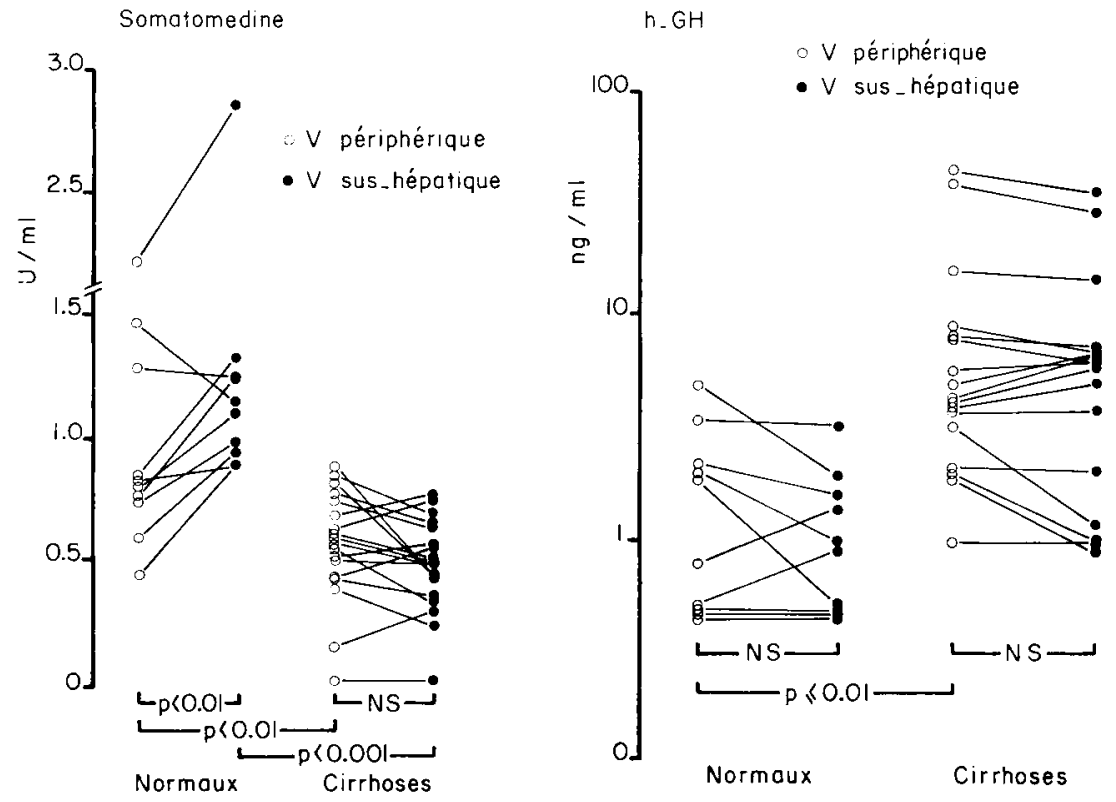

FIG. 1. - Activité somafomédine ef hormone de croissance dosées dans le sang périphérique ef dans le sang sus-hépatique chez 10 sujets normaux et 18 sujets atteints de cirrhose alcoolique.

\section{Conclusion.}

Chez les sujets atteints de cirrhose alcoolique étudiés ici, « l'activité SM » est abaissée ef l'hormone de croissance élevée, par rapport aux valeurs observées chez le sujet normal. Chez ces malades, et contrairement à ce qui est observé chez les témoins, il n'existe pas de différence significative entre « l'activité $S M$ » du sang sushépatique et celle du sang périphérique. Enfin chez les sujets atteints de cirrhose alcoolique, il existe une corrélation positive entre le DSH ef « l'activité SM » circulante.

Réunion Groupe Développement INRA/Productions animales Montpellier, 17-18 mai 1977.

\section{Références}

CAESAR J., SHALDON S., CHIANDUSSI L., GUEVARA L., SHERLOCK S., 1961. The use of indocyanine green in the measurements of hepatic blood flow and as a test of hepatic function. Clin. Sci., 21, 43-57. 
DaughadaY W. H., PHILliPS L. S., MUELLER M. C., 1976. The effects of insulin and growth hormone on the release of somatomedin by the isolated rat liver. Endocrinology, 98, 1214-1219.

DONNADIEU M., SCHIMPFF R. M., ALLAIRE Y., GIRARD F., 1967. Dosage radio-immunologique de l'hormone de croissance chez l'enfant. Path. Biol., 15, 865-872.

McCONAGHEY P. E., SLEDGE C. B., 1970. Production of sulphation factor by the perfused liver. Nature (Lond), 225, 1249-1250.

PHILLIPS L. S., HERINGTON A. C., KARL I. L., DAUGHADAY W. H., 1976. Comparison of somatomedin activity in perfusates of normal and hypophysectomized rats livers with and without added growth hormone. Endocrinology, 98, 606-614.

SCHIMPFF R. M., DONNADIEU M., 1973. Quantitative determination of somatomedin in human serum. Biomedecine, 14, 142-147.

UTHNE K., UTHNE T., 1972. Influence of liver resection and regeneration on somatomedin. Acta Endocr. (Kbh), 71, 255-264.

WU A., GRANT D. B., HAMBLEY J., LEVI A. J., 1974. Reduced serum somatomedin activity in patients with chronic liver disease. Clin. Sci. molec. Med., 47, 359-360. 